\title{
Optical surface modes in a system of fine metallic particles
}

\author{
A. Ya. Blank and L. V. Garanina \\ Radio Astronomy Institute, National Academy of Sciences of Ukraine, \\ 4 Krasnoznamennaya Str., 310002, Kharkov, Ukraine \\ E-mail: blank@ira.kharkov.ua \\ L. G. Grechko \\ Institute of Surface Chemistry, National Academy of Sciences of Ukraine, \\ 31 Nauki Ave., 252022, Kiev, Ukraine \\ E-mail: vit@surfchem.freenet.kiev.ua
}

Received December 14, 1998, revised April 6, 1999

\begin{abstract}
The interaction of electromagnetic radiation with a low-density disordered system of fine metallic particles located in a dielectric matrix is considered in a quasi-static approach with allowance for dipole-dipole interaction. The electric dipole moment of the system is calculated in a pair approximation. The case of nonidentical particles differing in their sizes or dielectric functions is presented. Allowance for particle nonidentity leads to doubling of the number of their surface modes and to the appearance of band gaps in the spectrum. The spectrum peculiarities are revealed in the fine structure of electromagnetic absorption.
\end{abstract}

PACS: 78.20.Ci

Examination of oscillation spectra of metals is of great importance in connection with the problem of investigation of their absorptive properties. The nonuniform metallic layers, whose thickness is small compared to the skin depth, demonstrate an anomalously large absorptivity. Thin metallic gratings possess a variety of unusual transmissive and reflective features in a wide frequency range [1]. A layer of fine metal particles soluted in a host dielectric absorbs nearly totally the radiation incident onto its surface [2]. These properties are attributed to the resonant excitation of the proper oscillation modes in a system and are very sensitive to the interaction between metal particles. Here we consider the last case - a small density system of disordered fine metal particles placed into a dielectric. The volume fraction of metal particles determined by the filling factor $f=4 / 3 \pi r^{3} n$, where $r$ is the particle size and $n$ is the particle density, is assumed to be small, $f<<1$ (in fact, $f \sim 0.1-0.2$ ). Because of this circumstance, we can restrict the discussion to only the pair interaction between metal particles which we consider as electric dipoles. This approach is developed in the theory of interacting hard sphere gas [3] and is commonly used to describe the vibrational spectra of a crystal lattice [4].

In this paper we study the interaction between nonidentical particles that differ in their sizes or dielectric properties (permittivities). This nonequivalence leads to the appearance of new spectral branches and band gaps. These results are similar to those found in one-dimensional vibrational chain of atoms when some of them are replaced by atoms of other kind [5,6]. It would be interesting to trace this analogy (and distinctions) in the case of infinite chain of metal particles with long-range multipole interaction. However, this will be the subject of another study.

The specific feature of the electromagnetic radiation (EMR) absorption in a system of fine metallic particles is a resonance caused by the existence of a surface mode (SM) in a single particle at the frequency [7]

$$
\omega_{s}=\frac{\omega_{p}}{\sqrt{\varepsilon_{\infty}+2 \varepsilon_{0}}} .
$$

Here it is assumed that a metal dielectric function has the form 


$$
\varepsilon(\omega)=\varepsilon_{\infty}-\frac{\omega_{p}^{2}}{\omega(\omega+i v)},
$$

where $\varepsilon_{\infty}$ is the lattice dielectric function; $\omega_{p}$ and $v$ are the electron plasma frequency and the collisional frequency, respectively; and $\varepsilon_{0}$ is the dielectric function of the environment.

This mode usually called Fröhlich's mode is related to uniform dipole electron oscillations in a particle [7]. In the electrostatic limit the polarization inside the particle is determined by well known relation [1]

$$
\mathbf{P}(\omega)=\frac{\varepsilon(\omega)-\varepsilon_{0}}{\varepsilon(\omega)+2 \varepsilon_{0}} r^{3} \mathbf{E}_{0},
$$

where $r$ is the particle radius and $\mathbf{E}_{0}$ is the external alternating electric field which we consider quasistatic. Using the dielectric function of a metal particle in the form (2), we can rewrite it in the form

$$
\mathbf{P}(\omega)=\left(\alpha_{\infty}+\frac{\omega_{s}^{2}\left(1-\alpha_{\infty}\right)}{\omega_{s}^{2}-\omega^{2}-i \omega v}\right) r^{3} \mathbf{E}_{0},
$$

where $\alpha_{\infty}=\left(\varepsilon_{\infty}-\varepsilon_{0}\right) /\left(\varepsilon_{\infty}+2 \varepsilon_{0}\right)$ is the Clausius-Mosotti factor. This expression clearly shows the resonance at the frequency of the surface mode. Thus, the interaction of an alternating field with a metallic particle results in oscillator excitation at the Fröhlich frequency $\omega_{s}$.

The EMR absorption in a system of metallic particles is sensitive to the magnitude and character of the direct multipole interaction between particles even though their concentration is sufficiently small [8-11].

This problem was analyzed theoretically by many authors [12-20]. In those studies alternative methods of calculating the effective dielectric parameters of the disperse systems containing different inclusions were developed. At the same time, comparatively not much attention was given to the investigation of the proper surface mode spectra in these systems. Even when considering a very simple system of two or three particles one finds there some peculiar singularities caused by multipole interaction between particles $[9,10,20]$

In this paper we investigate the spectrum of surface plasmons of two nonidentical metallic particles with different radii and different dielectric functions. In determining the polarization of the system with allowance for the interaction, we have used the results of the previous paper [11], where the calculation formalism for the effective dielectric

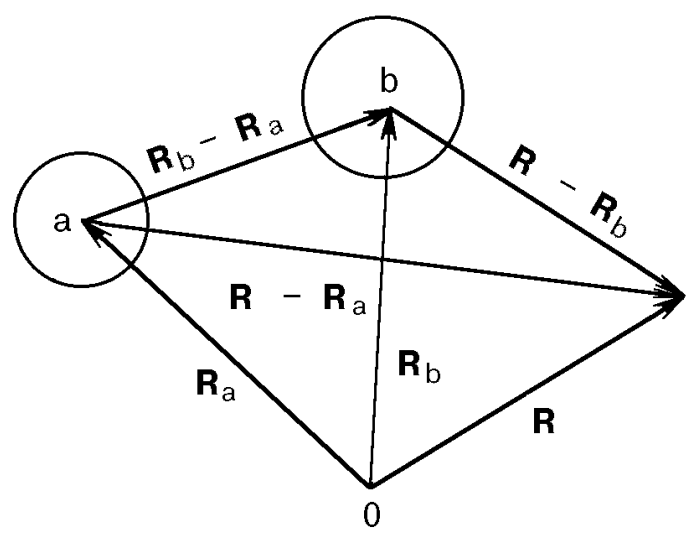

Fig. 1. Geometry of the particle location.

function of disperse systems with metallic inclusions was proposed. In present analysis we restrict the discussion to a very simple model:

1. The analysis is carried out in the long-wave approximation, i.e., the wavelengths are large compared with the size of the particle and the mean distance between them, $\lambda>>r, L$.

2 . The particle size is assumed greatly exceeds the electron mean free path, $r>>l$.

3 . The allowance for the interaction between particles is carried out in a pair approximation which we assume to be sufficient because of a small concentration of inclusions.

\section{The $N$-particles case}

Let us choose two particles $a$ and $b$ from the ensemble. We can now find the polarization of coupled particles with allowance for multipole interactions with other $N-2$ particles.

We define $\mathbf{R}_{a}$ as the radius-vector of the center of sphere $a, \mathbf{R}_{b}$ as the radius-vector of the center of sphere $b$, and $\mathbf{R}$ is an arbitrary point outside the spheres in the medium (see Fig. 1). In the case of a uniform external field $\mathbf{E}_{0}$, the potential inside sphere $a$ (which is regular at $\mathbf{R}=\mathbf{R}_{a}$ ) has the form [21]

$$
\varphi_{a}^{\text {in }}=-E_{0} \sum_{l, m} A_{l m}^{(a)}\left|\mathbf{r}-\mathbf{r}_{a}\right|^{l} Y_{l m}\left(\mathbf{R}-\mathbf{R}_{a}\right),
$$

where $\hat{\mathbf{R}} \hat{-} \mathbf{R}_{a}$ is an unit vector along $\mathbf{R}-\mathbf{R}_{a}$, and $Y_{l m}\left(\mathbf{R} \hat{-} \mathbf{R}_{a}\right)$ are spherical harmonics. The potentials at the point $\mathbf{R}$ outside sphere $a$ can be represented in the form

$$
\begin{gathered}
\varphi_{a}^{\text {out }}=-E_{0} \sum_{l, m} d_{l m}\left|\mathbf{R}-\mathbf{R}_{a}\right|^{l} Y_{l m}\left(\mathbf{R} \hat{-} \mathbf{R}_{a}\right)- \\
-E_{0} \sum_{l, m} B_{l m}^{(a)}\left|\mathbf{R}-\mathbf{R}_{a}\right|^{-l-1} Y_{l m}\left(\mathbf{R} \hat{-} \mathbf{R}_{a}\right)- \\
-E_{0} \sum_{b \neq a} \sum_{l, m} B_{l, m}^{(b)}\left|\mathbf{R}-\mathbf{R}_{b}\right|^{-l-1} Y_{l, m}\left(\left|\mathbf{R} \hat{-} \mathbf{R}_{b}\right|\right),
\end{gathered}
$$


where the first term is the potential of the external field, the second term is the potential created by the particle $a$, and the third term includes the effect of the remaining particles.

The potentials (4) and (5) must satisfy the standard boundary conditions at the surface of sphere $a$ :

$$
\begin{gathered}
\left.\varphi_{a}^{\text {in }}\right|_{\mathbf{R}=\mathbf{R}_{s a}}=\left.\varphi_{a}^{\text {out }}\right|_{\mathbf{R}=\mathbf{R}_{s a}} \\
\varepsilon_{a}\left(\nabla \varphi_{a}^{\text {in }} \mathbf{n}_{a}\right)_{\mathbf{R}=\mathbf{R}_{s a}}=\varepsilon_{0}\left(\nabla \varphi_{a}^{\text {out }} \mathbf{n}_{a}\right)_{\mathbf{R}=\mathbf{R}_{s a}} .
\end{gathered}
$$

Accordingly, we must reduce the expressions for the potentials to a single center of the sphere, which can be done with use of the following transformation of tesseral harmonics [22-24]:

$$
\begin{gathered}
\frac{Y_{l, m}\left(\mathbf{R}-\mathbf{R}_{b}\right)}{\left|\mathbf{R}-\mathbf{R}_{b}\right|^{l+1}}= \\
=\sum_{l^{\prime}, m^{\prime}} Q_{l^{\prime}, m^{\prime}}^{l, m}\left(\mathbf{R}_{b}-\mathbf{R}_{a}\right) \mid \mathbf{R}-\mathbf{R}_{a} l^{l^{\prime}} Y_{l^{\prime}, m^{\prime}}\left(\mathbf{R} \hat{-} \mathbf{R}_{a}\right),
\end{gathered}
$$

where

$$
\begin{gathered}
Q_{l^{\prime}, m^{\prime}}^{l, m}\left(\mathbf{R}_{b}-\mathbf{R}_{a}\right)=(-1)^{l+m} \frac{Y_{l+l^{\prime}, m^{\prime}-m}^{*}\left(\mathbf{R}_{b} \hat{-} \mathbf{R}_{a}\right)}{\left|\mathbf{R}_{b}-\mathbf{R}_{a}\right|^{l^{\prime}+l+1}} \times \\
\times \frac{4 \pi(2 l+1)\left(l+l^{\prime}+m-m^{\prime}\right) !\left(l^{\prime}+l+m^{\prime}-m\right) !}{\left(2 l^{\prime}+1\right)\left(2 l+2 l^{\prime}+1\right)(l+m) !(l-m) !\left(l^{\prime}-m^{\prime}\right) !\left(l^{\prime}+m^{\prime}\right) !},
\end{gathered}
$$

where $\left|\mathbf{R}-\mathbf{R}_{a}\right|<\left|\mathbf{R}_{b}-\mathbf{R}_{a}\right|$.

After putting expressions (4) and (5) in the boundary conditions (6) and (7) and using (8), we obtain a system of equations for the coefficients $B_{l, m}^{(a)}$ :

$$
\frac{B_{l, m}^{(a)}}{\alpha_{l}^{(a)}}+\sum_{b \neq a} \sum_{l^{\prime}, m^{\prime}} B_{l^{\prime}, m^{\prime}}^{(b)} Q_{l, m}^{l^{\prime}, m^{\prime}}\left(\mathbf{R}_{b}-\mathbf{R}_{a}\right)=-d_{l, m} .
$$

Equation (9), in principle, gives the general solution for finding the polarization of a single particle $\left(B_{\mathrm{l} m}^{(a)}\right)$ with allowance for the multipole interactions with other particles.

In the case of two particles $(a=i ; b=j)$ we obtain the following expressions from (9):

$$
\begin{gathered}
\frac{B_{l, m}^{(i)}+\sum_{l}^{(i)}}{\alpha_{l}, m^{\prime}}\left[B_{l^{\prime}, m^{\prime}}^{(j)}(-1)^{m^{\prime}+l^{\prime}} \times\right. \\
\left.\times \frac{Y_{l+l^{\prime}, m-m^{\prime}}\left(\mathbf{R}_{j} \hat{-} \mathbf{R}_{i}\right)}{\mathbf{R}_{i j}^{l+l^{\prime}+1}} K_{l, m}^{l^{\prime}, m^{\prime}}\right]=-d_{l, m},
\end{gathered}
$$

$$
\begin{gathered}
\frac{B_{l, m}^{(j)}}{\alpha_{l}^{(j)}}+\sum_{l^{\prime}, m^{\prime}}\left[B_{l^{\prime}, m^{\prime}}^{(i)}(-1)^{m^{\prime}+l^{\prime}} \times\right. \\
\left.\times \frac{Y_{l+l^{\prime}, m-m^{\prime}}\left(\mathbf{R}_{j} \hat{-} \mathbf{R}_{i}\right)}{\mathbf{R}_{i j}^{l+l^{\prime}+1}} K_{l, m}^{l^{\prime}, m^{\prime}}\right]=-d_{l, m} .
\end{gathered}
$$

Here we define

$$
K_{l, m}^{l^{\prime}, m^{\prime}}=\left[\frac{4 \pi\left(2 l^{\prime}+1\right)\left(l+l^{\prime}+m-m^{\prime}\right) !\left(l+l^{\prime}+m^{\prime}-m\right) !}{(2 l+1)\left(2 l+2 l^{\prime}+1\right)(l+m) !(l-m) !\left(l^{\prime}+m^{\prime}\right) !\left(l^{\prime}-m^{\prime}\right) !}\right]^{1 / 2}
$$

and $\alpha_{l}^{(i)}=l\left(\varepsilon_{i}-\varepsilon_{0}\right) r_{i}^{2 l+1} /\left[l \varepsilon_{i}+(l+1) \varepsilon_{0}\right]$, where $R_{i j}=\left|\mathbf{R}_{j}-\mathbf{R}_{i}\right|$ and $r_{i}$ is the radius of the particle $i$.

We will discuss only the case of the dipole-dipole interaction $\left(l=l^{\prime}=1\right)$. We choose the axis $Z$ along the vector $\left(\mathbf{R}_{j}-\mathbf{R}_{i}\right)$. From (5) and (10) we then obtain the dipole moment of the particle $i$ [11]:

$$
\begin{aligned}
& \mathbf{p}_{i}(i) \cdot \mathbf{m}=-\mathbf{E}_{0} \mathbf{m} \sum_{m=-1}^{1} B_{1 m}^{(i)} Y_{1 m}\left(\mathbf{R}-\mathbf{R}_{i}\right)= \\
& =\left[X_{10}^{(i)} n_{z} m_{z}+X_{11}^{(i)}\left(n_{x} m_{x}+n_{y} m_{y}\right)\right] E_{0},
\end{aligned}
$$

where $\mathbf{m}=\left(\mathbf{R}-\mathbf{R}_{i}\right) / \mathbf{R}-\mathbf{R}_{i} \mid, \mathbf{n}=\mathbf{E}_{0} / E_{0}$, and

$$
\begin{gathered}
X_{10}^{(i)}=\alpha_{1}^{(j)} \frac{1+2 \alpha_{1}^{(j)} R_{i j}^{-3}}{1-4 \alpha_{1}^{(i)} \alpha_{1}^{(j)} R_{i j}^{-6}}, \\
X_{11}^{(i)}=\alpha_{1}^{(i)} \frac{1-\alpha_{1}^{(j)} R_{i j}^{-3}}{1-\alpha_{1}^{(i)} \alpha_{1}^{(j)} R_{i j}^{-6}}
\end{gathered}
$$

are the longitudinal and transverse polarizabilities, respectively, and $\alpha_{1}^{(i)}=\left(\varepsilon_{i}-\varepsilon_{0}\right) r_{i}^{3} /\left(\varepsilon_{i}+2 \varepsilon_{0}\right)$ is the polarizability of an isolated single particle. 


\section{Surface modes in a system of two particles}

To obtain the spectrum of surface modes we represent the expressions (13) and (14) in a convenient form

$$
\begin{aligned}
& X_{10}=\frac{A_{2}(\omega)+2 \Delta^{3} \sigma_{1}^{3}}{A_{1}(\omega) A_{2}(\omega)-4 \Delta^{3} \sigma_{1}^{6}} r_{1}^{3}, \\
& X_{11}=\frac{A_{2}(\omega)-\Delta^{3} \sigma_{1}^{3}}{A_{1}(\omega) A_{2}(\omega)-\Delta^{3} \sigma_{1}^{6}} r_{1}^{3},
\end{aligned}
$$

where $\quad \Delta=r_{2} / r_{1}, \quad \sigma_{1}=r_{1} / R, \quad$ and $\quad A_{i}(\omega)=$ $=\left[\varepsilon_{i}(\omega)+2 \varepsilon_{0}\right] /\left[\varepsilon_{i}(\omega)-\varepsilon_{0}\right](i=1,2)$.

The spectrum of surface modes is determined by the polarizability singularities, i.e., from the following relations:

$$
A_{1}(\omega) A_{2}(\omega)-4 \Delta^{3} \sigma_{1}^{6}=0
$$

and

$$
A_{1}(\omega) A_{2}(\omega)-\Delta^{3} \sigma_{1}^{6}=0
$$

for the longitudinal and transverse modes, respectively.

Relations (17) and (18) determine the frequencies of the electron homogeneous oscillations of particles of different sizes and different dielectric functions.

2.1. In the case of the same particles $\left(r_{1}=r_{2}=r\right.$, $\varepsilon_{1}=\varepsilon_{2}=\varepsilon$ ) we have $\Delta=1, A_{1}(\omega)=A_{2}(\omega)$, the extra poles in expressions (15) and (16) are cancelled, and we obtain the two known surface modes [10]: for the longitudinal mode

$$
\omega_{\|}^{2}=\omega_{s}^{2} \frac{1-2(r / R)^{3}}{1-2(r / R)^{3} \alpha_{\infty}},
$$

where $\omega_{s}$ is Fröhlich's surface mode defined by (1), and for the transverse surface mode defined from (18), which is

$$
\omega_{\perp}^{2}=\omega_{s}^{2} \frac{1+(r / R)^{3}}{1+(r / R)^{3} \alpha_{\infty}} .
$$

We see, therefore, that allowance for the dipoledipole interaction between particles in the presence of an external alternating field leads to doubling the proper mode number - to the appearance of longitudinal and transverse modes.

Note that in the case of identical particles with arbitrary orientation of the external field the dipole interaction between particles shifts the longitudinal mode to low-frequency side and the transverse mode to high-frequency side. These shifts are nonsymmetrical that lead to some absorption peculiarities in the many-particle system [see Eq. (10) in Ref. 11].

Let us now consider the changes in the surface mode spectrum caused by the nonidentity of the particles.

2.2. Let us consider particles of different sizes $(\Delta \neq 1)$ but with the same dielectric properties $\left(\varepsilon_{1}=\varepsilon_{2}\right)$. In this case from Eqs. (17) and (18) we obtain, in contrast with the case of identical particles, the four surface modes instead of two:

$$
\begin{aligned}
& \omega_{\|}^{2}=\omega_{s}^{2} \frac{1-2 \Delta^{3 / 2}\left(r_{1} / R\right)^{3}}{1-2 \Delta^{3 / 2}\left(r_{1} / R\right)^{3} \alpha_{\infty}}, \\
& \tilde{\omega}_{\|}^{2}=\omega_{s}^{2} \frac{1+2 \Delta^{3 / 2}\left(r_{1} / R\right)^{3}}{1+2 \Delta^{3 / 2}\left(r_{1} / R\right)^{3} \alpha_{\infty}}, \\
& \omega_{\perp}^{2}=\omega_{s}^{2} \frac{1+\Delta^{3 / 2}\left(r_{1} / R\right)^{3}}{1+\Delta^{3 / 2}\left(r_{1} / R\right)^{3} \alpha_{\infty}}, \\
& \tilde{\omega}_{\perp}^{2}=\omega_{s}^{2} \frac{1-\Delta^{3 / 2}\left(r_{1} / R\right)^{3}}{1-\Delta^{3 / 2}\left(r_{1} / R\right)^{3} \alpha_{\infty}} .
\end{aligned}
$$

We see from these formulas that allowance for the interaction between particles with different sizes leads to splitting of the longitudinal and transverse frequencies, which are shifted (for longitudinal and transverse modes) to the opposite sides. The dependence of the proper frequencies on the particle size ratio is shown in Fig. 2. Note that by setting $\Delta=1$, i.e., passing to the case of identical particles, the extra modes $\tilde{\omega}_{\|}$and $\tilde{\omega}_{\perp}$ formally do not disappear. The point is that the oscillator forces corresponding to these modes turn to zero when $\Delta=1$ [see below Eqs. (30) and (31)]. The situation considered here reminds one of the appearance of an

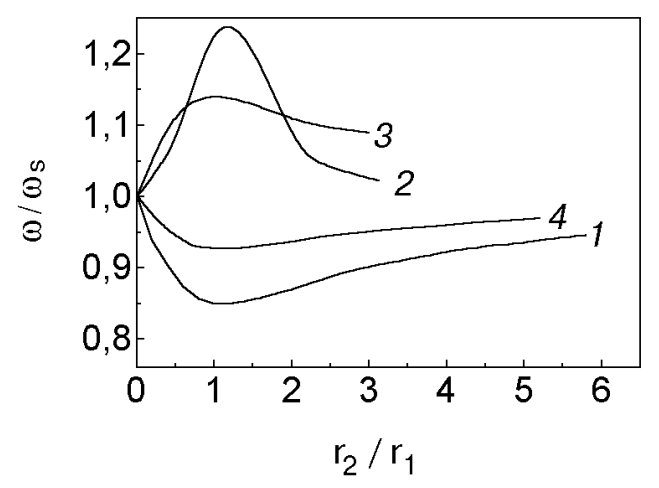

Fig. 2. Dependence of proper frequencies on the particle size ratio: $1-\omega_{\|} / \omega_{s} ; 2-\tilde{\omega}_{\|} / \omega_{s} ; 3-\omega_{\perp} / \omega_{s} ; 4-\tilde{\omega}_{\perp} / \omega_{s}$. 


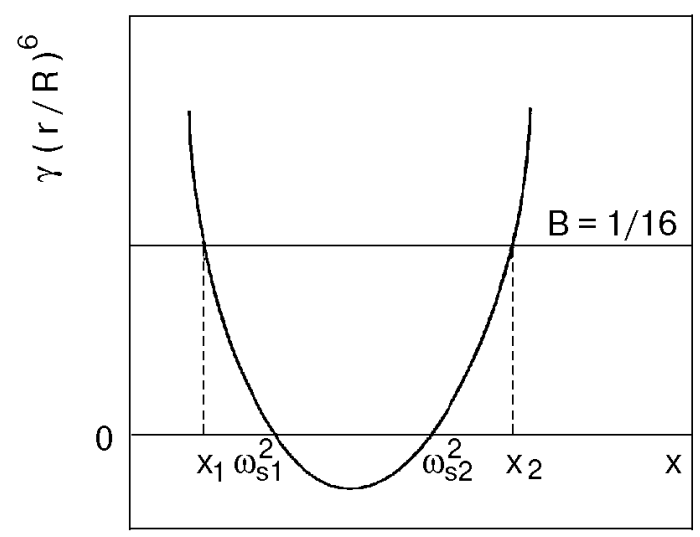

Fig. 3. Graphic solution of Eq. (29) for $\Gamma>1$.

optical mode in the one-dimensional infinite chain of two kinds of atoms with different masses in the unit cell.

2.3. Let us examine now another limiting case of particles with the same sizes but with different dielectric properties $\left(\varepsilon_{1} \neq \varepsilon_{2}\right)$. In this case Eqs. (17) and (18) give the following relations for finding the surface modes:

for the longitudinal mode

$$
\frac{\varepsilon_{1}(\omega)+2 \varepsilon_{0}}{\varepsilon_{1}(\omega)-\varepsilon_{0}} \frac{\varepsilon_{2}(\omega)+2 \varepsilon_{0}}{\varepsilon_{2}(\omega)-\varepsilon_{0}}=4\left(\frac{r}{R}\right)^{6}
$$

for the transverse mode

$$
\frac{\varepsilon_{1}(\omega)+2 \varepsilon_{0}}{\varepsilon_{1}(\omega)-\varepsilon_{0}} \frac{\varepsilon_{2}(\omega)+2 \varepsilon_{0}}{\varepsilon_{2}(\omega)-\varepsilon_{0}}=\left(\frac{r}{R}\right)^{6} .
$$

Using Eq. (2), we can write these relations in the form (here we set $\varepsilon_{0}=\varepsilon_{\infty}=1$ )

$$
\left(\omega^{2}-\omega_{s 1}^{2}\right)\left(\omega^{2}-\omega_{s 2}^{2}\right) \frac{1}{\omega_{s 1}^{2} \omega_{s 2}^{2}}=\gamma\left(\frac{r}{R}\right)^{6},
$$

where

$$
\omega_{s 1,2}=\frac{\omega_{p 1,2}}{\sqrt{3}}
$$

are the proper modes of surface oscillations of the separated spheres; $\gamma=4$ or 1 in the case of the longitudinal or transverse mode, respectively.

Introducing a variable $x=\omega^{2} / \omega_{s 1}^{2}$, we transform (27) to the form

$$
(x-1)(\Gamma x-1)=\gamma\left(\frac{r}{R}\right)^{6} \equiv B, \quad \Gamma=\left(\frac{\omega_{p 1}}{\omega_{p 2}}\right)^{2} .
$$

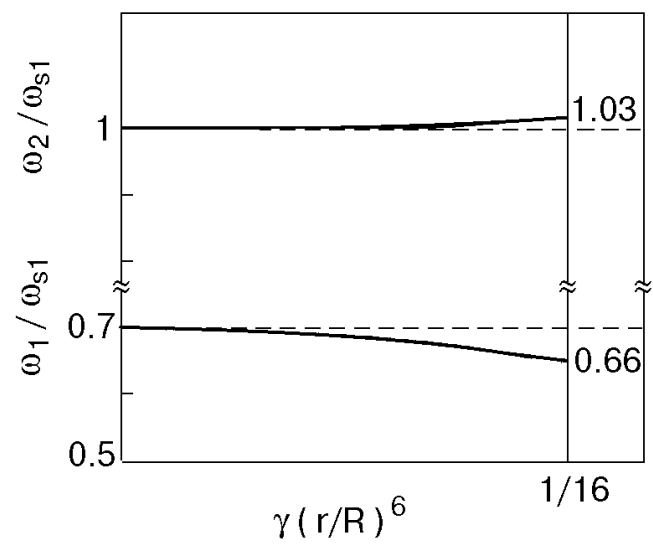

Fig. 4. Frequency spectrum versus the distance between particles.

The graphical solution of (29) for $\Gamma>1$ is shown in Fig. $3 ; x_{1}$ and $x_{2}$ are the roots of Eq. (29). We see in this figure that the frequency region between $\omega_{p 1}^{2} / 3$ and $\omega_{p 2}^{2} / 3$ creates the band gap. For the longitudinal mode $(\gamma=4)$, when the distance between particles is $R=2 r$, we have $x_{1}=0.22$ and $x_{2}=1.02$. In this case the analogy with the appearance of band gaps in the one-dimensional vibrational chain is appropriate.

The dependence of the proper frequencies on the distance between particles is shown in Fig. 4.

The general case of nonequivalent spheres $\left(r_{1} \neq r_{2}, \varepsilon_{1} \neq \varepsilon_{2}\right)$ will be reported elsewhere.

\section{Discussion of the results}

As is shown in Sec. 2, allowance for the particle nonidentity leads to doubling of the number of proper modes in a system of two spheres. Two supplementary modes, analogous with the optical modes in the one-dimensional lattices, appear together with two known surface modes for equivalent spheres. The longitudinal and transverse parts of the polarization, in accordance with expressions (15) and (16), can be represented in the form

$$
\begin{gathered}
X_{10}=\frac{1}{2} r_{1}^{3}\left[\frac{1+\Delta^{3 / 2}}{A(\omega)-2 \Delta^{3 / 2} \sigma_{1}^{3}}+\frac{1-\Delta^{3 / 2}}{A(\omega)+2 \Delta^{3 / 2} \sigma_{1}^{3}}\right], \\
X_{11}=\frac{1}{2} r_{1}^{3}\left[\frac{1-\Delta^{3 / 2}}{A(\omega)-\Delta^{3 / 2} \sigma_{1}^{3}}+\frac{1+\Delta^{3 / 2}}{A(\omega)+\Delta^{3 / 2} \sigma_{1}^{3}}\right\rfloor,
\end{gathered}
$$

where the poles of the denominators correspond to new modes. The factors $1 \pm \Delta^{3 / 2}$ are oscillator forces which characterize the appropriate oscillations. All the expressions entering into Eq. (30), 
[Eq. (31), respectively], can be represented in the appropriate form, for instance

$$
\begin{gathered}
\frac{1+\Delta^{3 / 2}}{A(\omega)-2 \Delta^{3 / 2} \sigma_{1}^{3}}= \\
=\frac{\left(1+\Delta^{3 / 2}\right)}{(1-A)\left(1-\alpha_{\infty} A\right)}\left(\alpha_{\infty}(1-A)+\frac{\omega_{\|}^{2}\left(1-\alpha_{\infty}\right)}{\omega_{\|}^{2}-\omega^{2}-i \omega \nu}\right),
\end{gathered}
$$

where $\omega_{\|}$is given by (21) and $A=2 \Delta^{3 / 2}\left(r_{1} / R\right)^{3}$.

We see, therefore, that the response of a system of two nonidentical particles is given by the sum of responses corresponding to four independent oscillators; their frequencies are the proper modes of the system (21)-(24).

The same is also true in the case of spheres of equal size but with different dielectric functions.

We appreciate the valuable discussion with E. Syrkin of the analogy of the considered spectra with the vibrational ones.

1. Surface Excitations, V. M. Agranovich and D. L. Mills (eds.), Amsterdam, North Holland (1985).

2. A. Ya. Blank and A. Ya. Sharshanov, Fiz. Nizk. Temp. 21, 336 (1995) [Low. Temp. Phys. 21, 256 (1995)].

3. R. Balesku, Equilibrium and Nonequilibrium Statistical Mechanics, Wiley (1975).

4. A. M. Kosevich, Theory of Crystal Lattice, Vyscha shkola, Kharkov (1988)

5. F. G. Bass, V. L. Fal'ko, and S. I. Khankina, Ukr. Phys. J. 38, 901 (1993).
6. M. A. Mamalui, E. S. Syrkin, and S. B. Feodosjev, Fiz. Nizk. Temp. 24, 773 (1998) [Low. Temp. Phys. 24, 583 (1998)].

7. C. F. Bohren and D. P. Huffman, Absorption and Scattering of Light by Small Particles, Wiley and Sons (1983).

8. U. Kreibig and M. Vollmer, Optical Properties of Metal Clasters, Springer Series in Material Science 25, Springer (1995).

9. R. Ruppin, Phys. Rev. B26, 3440 (1982).

10. M. Inoue and K. Ohtaka, J. Phys. Soc. Jpn. 52, 3853 (1983).

11. L. G. Grechko, A. Ya. Blank, V. V. Motrich, A. A. Pinchuk, and L. V. Garanina, Radio Phys. and Radio Astron. 2, 19 (1997).

12. A. Liebsch and B. N. Persson, J. Phys. C16, 5375 (1983).

13. R. G. Barrera, G. Monsivais, W. L. Mochan, and E. Anda, Phys. Rev. B39, 1989 (1989).

14. V. A. Davis and L. Schwartz, Phys. Rev. B31, 6627 (1986).

15. B. U. Felderholf, G. W. Ford, and E. G. D. Cohen, J. Stat. Phys. 28, 649 (1982).

16. B. U. Felderholf, G. W. Ford, and E. G. D. Cohen, J. Stat. Phys. 28, 135 (1982).

17. W. Lamb, D. M. Wood, and N. W. Aschcroft, Phys. Rev. B21, 2248 (1980).

18. L. Fu and L. Resca, Phys. Rev. B49, 6625 (1984).

19. P. Clippe, R. Evrard, and A. A. Lucas, Phys. Rev. B14, 1715 (1976).

20. J. M. Gerardy and M. Ausloos, Phys. Rev. B25, 4204 (1982).

21. P. M. Morse and H. Feshbach, Methods of Theoretical Physics, McGraw-Hill Book Company, Inc. (1953).

22. M. Danos and L. C. Maximon, J. Math. Phys. 6, 766 (1965).

23. O. R. Crusan, Quart. Appl. Math. 20, 33 (1962).

24. R. Nozawa, J. Math. Phys. 7, 1841 (1966). 